\section{Hipatia Press}

www.hipatiapress.com

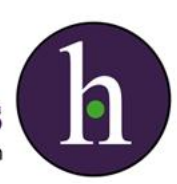

Instructions for authors, subscriptions and further details:

http://rise.hipatiapress.com

\title{
El Impacto del Bono Demográfico en el Sector Educativo Argentino. Periodo 2010-2040. Un Abordaje Interdisciplinario
}

Fernando Ariel Manzano ${ }^{1}$

1) Universidad Nacional de Córdoba, Argentina

Date of publication: October $25^{\text {th }}, 2015$

Edition period: October 2015-February 2016

To cite this article: Manzano, F.A. (2015). El Impacto del Bono Demográfico en el Sector Educativo Argentino. Periodo 2010-2040. Un Abordaje Interdisciplinario. International Journal of Sociology of Education, 4(3), 225-256. doi: 10.17583/rise.2015.1692

To link this article: http://dx.doi.org/10.17583/rise.2015.1692

\section{PLEASE SCROLL DOWN FOR ARTICLE}

The terms and conditions of use are related to the Open Journal System and to Creative Commons Attribution License (CC-BY) 


\section{The Impact of the Demographic Dividend in the Argentine Education Sector. Period 2010-2040. An Interdisciplinary Approach}

Fernando Ariel Manzano

Universidad Nacional de Córdoba

(Received: 19 August 2015; Accepted: 6 October 2015; Published: 25

October 2015)

\section{Abstract}

As the demographic transition progresses there is a period of sustained fertility decline, meanwhile it is not very large relative weight of the adult population and, therefore, the major proportion of the population is concentrated in the ages potentially active. From the demographic theory this period is noted for its advantages to boost economic growth. However, since economic theory, it is noted that the necessary condition for success of the demographic dividend, is to raise levels of activity rates and employment. Namely to ensure a scenario of economic growth, implying, reverse causality proposed by the demographic theory. Given the interrelationship between population and economic dynamics, the objective of this study is to quantify the impact of declining dependency burden of the education sector in economic terms. In order to do this, you must make a number of assumptions that allow us to depend on the evolution of expenditure on education and GDP growth of only changes in the age structure of the population Argentina during the period 2010-2040, which It unfolds as the demographic transition unfolds.

Keywords: demographic bonus, education, Argentina, transfer. 


\section{El Impacto del Bono Demográfico en el Sector Educativo Argentino. Periodo 2010-2040. Un Abordaje Interdisciplinario}

Fernando Ariel Manzano

Universidad Nacional de Córdoba

(Recibido: 19 de Agosto 2015; Aceptado: 6 de Octubre 2015; Publicado: 25 Octubre 2015)

\section{Resumen}

Conforme avanza la transición demográfica, existe un periodo de tiempo de descenso sostenido de la fecundidad, mientras que aún no es muy grande el peso relativo de la población mayor de edad y, por tanto, la principal proporción de la población se concentra en las edades potencialmente activas.Desde la teoría demográfica se destaca este periodo por sus ventajas para potenciar el crecimiento económico. Sin embargo, desde la teoría económica, se advierte que la condición necesaria para que tenga éxito el bono demográfico, es elevar los niveles de las tasas de actividad y de empleo. Es decir, garantizar un escenario de crecimiento económico, lo cual implicaría, invertir la causalidad propuesta por la teoría demográfica. Teniendo en cuenta la interrelación entre la dinámica demográfica y económica, el objetivo de este trabajo es cuantificar el impacto de la disminución de la carga de dependencia del sector educativo, en términos económicos. Para poder realizarlo es necesario efectuar una serie de supuestos, que nos permitan hacer depender la evolución del gasto en educación y el crecimiento del PBI, de solamente los cambios en la estructura de edad de la población Argentina durante el periodo 2010-2040, que se desenvuelve conforme se desarrolla la transición demográfica.

Palabras clave: bono demográfico, educación, Argentina, transferencia. 
egún el diccionario demográfico de Naciones Unidas (1959) se define a la demografía como: "una ciencia que tiene como finalidad el estudio de la población humana y que se ocupa de su dimensión, estructura, evolución y caracteres generales considerados fundamentalmente desde un punto de vista cuantitativo". Así entendida, la demografía se dedica a estudiar de forma estadística las conductas humanas en términos de sus variables fundamentales, la fecundidad, la mortalidad y la migración. La teoría más utilizada dentro de las ciencias demográficas para describir el cambio demográfico de las poblaciones es la llamada "teoría de la transición demográfica" (TTD). Sus planteos básicos proponen una descripción de la evolución de la mortalidad, la natalidad y el crecimiento demográfico. Esta teoría se originó para definir y describir los cambios de la población, sobre todo en Europa (Zavala de Cosio, 1995).

El uso de la misma es útil como referencia del proceso de cambio en la estructura de edades de las poblaciones. Un análisis empírico, utilizando datos poblacionales históricos, demuestra que los cambios demográficos de muchos países están lejos de poder adaptarse a la regularidad propuesta por la TTD -sobre todo en ciertos países de América Latina y el Caribe, en los que se destaca una importante participación del componente migratorio(Manzano, 2015).

En la actualidad la Argentina se encuentra atravesando un período en que la proporción de personas en edades potencialmente productivas (PET) crece de manera sostenida en relación a la de personas en edades potencialmente inactivas. Lo que en términos demográficos indicaría un descenso de la carga económica sobre los potenciales activos.

Dentro del campo de las ciencias demográficas, a partir de las dos últimas décadas del siglo pasado, comienzan a cobrar notoriedad un número importante de trabajos empíricos que afirman que la estructura de edad de la población (no su tamaño, ni su crecimiento como se había considerado en el pasado) es una variable que afecta significativamente al crecimiento económico, destacándose el intervalo de tiempo en donde se produce un crecimiento de la proporción de la población comprendida en las edades activas, a este periodo se lo denominó como bono demográfico. En el caso de la Argentina el fin del periodo del bono demográfico sería en el año 2035.

Sin embargo no debemos dejar de lado que dentro de la teoría económica 
se han propuesto interpretaciones diversas y muchas veces contradictorias respecto de las principales causas acerca de cómo generar el crecimiento económico, y con el paso del tiempo se ha hecho cada vez más difícil lograr cierto consenso debido a la proliferación de diferentes argumentaciones que se fueron desarrollando de manera independiente dentro de estas diversas escuelas económicas (Manzano, 2015).

En síntesis, los usos que hacen la demografía y la economía sobre el concepto del crecimiento económico, se encuentran totalmente desconectados. Un primer paso para interrelacionar ambas disciplinas, es llevar adelante la difícil tarea de articular diferentes marcos metodológicos, por tanto, todo avance en este camino representa un aporte al conocimiento social.

Utilizando el enfoque de las Cuentas Nacionales de Transferencias (CNT), que permite integrar las tendencias demográficas con ciertos instrumentos claves de la economía, nos proponemos llevar adelante el objetivo de este artículo. El mismo consiste en cuantificar en términos económicos, la reducción del peso demográfico del sector educativo, producto de los cambios que se producen en la estructura de edad, debido al avance de la transición demografía en Argentina durante el periodo 20102040

Los insumos que utilizaremos son los resultados del último censo Nacional de Población del año 2010 en Argentina, las proyecciones de población para el total del país, por sexo y en edades simples -que surgen a partir de los resultados del Censo Nacional 2010- y la información de determinados agregados económicos que surgen del sistema de Cuentas Nacionales base 2004. Por tanto, la información tendrá una única fuente que es el Instituto Nacional de Estadística y Censos (INDEC) de Argentina.

\section{Algunas Consideraciones Metodológicas}

La incorporación del enfoque de las Cuentas Nacionales de Transferencias (CNT), permite integrar las tendencias demográficas con ciertos instrumentos claves de la economía, para dar cuenta de los cambios en los tamaños de los grupos etarios y en sus necesidades cambiantes a través del tiempo. En términos generales, durante la infancia y la vejez las personas consumen más de lo que producen, mientras que durante ciertos años de la 
adultez la mayoría de las personas son productores netos.

La teoría más utilizada dentro de las ciencias demográficas para describir el cambio demográfico de las poblaciones es la llamada "teoría de la transición demográfica" ${ }^{2}$ (TTD). Sus planteos básicos proponen una descripción de la evolución de la mortalidad, la natalidad y el crecimiento demográfico. Esta teoría se originó para definir y describir los cambios de la población, sobre todo en Europa y en Estados Unidos, y además avanza en la búsqueda de las interrelaciones de la dimensión demográfica con el campo socioeconómico, lo que plantea diversos problemas y limitaciones, entre otros aspectos, en relación con los inicios y finales de la transición; la velocidad y ritmo del proceso; la interacción entre los factores demográficos; la uniformidad de la transición al interior de los países; la modernización económica; la urbanización y la diversidad étnica (Benítez Zenteno, 2004).

En este artículo no llevaremos adelante una crítica hacia los supuestos sobre los que se basa la TTD, sólo mencionaremos que el uso de la misma únicamente es útil en términos de una descripción general y de largo plazo, en donde se destacan los cambios en los pesos relativos en los grupos de edades, en las distintas generaciones.

Un análisis empírico de un periodo extendido en el tiempo utilizando variables demográficas, demuestra que los cambios demográficos de muchos países están lejos de poder adaptarse a la regularidad propuesta por la TTD sobre todo en ciertos países de América Latina y el Caribe, en los que se destaca una importante participación del componente migratorio- (Manzano, 2015).

Dentro del campo de las ciencias demográficas, a partir de las dos últimas décadas del siglo pasado, cobran notoriedad los trabajos empíricos que afirman que la estructura de edad de la población (no su tamaño, ni su crecimiento como se había considerado en el pasado) es una variable que afecta significativamente al crecimiento económico, destacándose el intervalo de tiempo en donde se produce un crecimiento de la proporción de la población comprendida en las edades activas, definiendo a este periodo como bono demográfico (Cendejas Bueno y Roa García, 2007, p.6-11).

El llamado bono demográfico fue acuñado por David Bloom en el año 1998 para hacer referencia a la etapa de la TTD en la cual, debido al descenso sostenido de la fecundidad, la población infantil y juvenil se ha reducido sustancialmente en términos relativos, mientras que aún no es muy 
grande el peso relativo de la población mayor de edad sobre el total poblacional y, por tanto, la principal proporción de población se concentra en las edades activas (Aguirre Pinto, 2011). Como consecuencia, las relaciones de dependencia demográfica (el cociente entre la población potencialmente inactiva y la población potencialmente activa), desciende hasta alcanzar sus niveles más bajos, momento en que finaliza el bono demográfico.

Los defensores del bono demográfico afirman que durante este periodo, los países disponen de una oportunidad de mejoramiento económico y social sin precedentes. (Saad et al., 2012)

Tanto las relaciones de dependencia demográfica, como el periodo correspondiente al bono demográfico, solo hacen referencia a relaciones entre variables de naturaleza demográfica. Pero para poder generar estos beneficios económicos, es necesario incrementar los niveles de las tasas de actividad y empleo - sin que se produzcan descensos en los niveles de productividad-. Debido a que el primer efecto del bono demográfico es incrementar la presión sobre el mercado laboral, lo que podría conducir a un aumento del desempleo o a un descenso del nivel de los salarios -o cualquier combinación de entre ambas-. En síntesis, el éxito del bono demográfico depende de que esté garantizado un escenario de crecimiento económico, lo cual implica, a la inversa de la propuesta de los defensores del bono demográfico, que puede ser una restricción más al crecimiento económico.

Dentro de las ciencias económicas no existe ninguna certeza acerca de cuáles son los factores determinantes respecto al crecimiento económico. En su desarrollo se destaca la proliferación de interpretaciones diversas y muchas veces contradictorias entre los diferentes paradigmas (Castro Barrios, 2005). Algunos se caracterizan por su excesiva abstracción que permite la coexistencia de factores explicativos no siempre coincidentes y mientras otros, se empeñan en explicar determinantes muy diversos sobre el crecimiento económico (Corrales, 1992, p.41).

A continuación, realizaremos un breve recorrido sobre la diversidad de posturas en la evolución del conocimiento sobre el crecimiento económico dentro de la teoría económica.

Los primeros análisis vinculados al crecimiento económico provienen de la época de los economistas clásicos. A mediados del siglo XVIII, el modelo elaborado por Adam Smith (1723-1790), y luego cuestionado por Malthus 
(1798) tenía un substrato esencialmente agrarista, propio del contexto de su época. La existencia de tierras libres, generaba que ante un exceso de población, las vías de escape fueran la emigración y la roturación de nuevas tierras, por tanto no había restricciones al crecimiento. Pero llegado el momento en que todas las tierras fértiles quedaran ocupadas, las nuevas tierras proporcionarían menos producción para una misma cantidad de trabajo (lo que se denomina dentro de la teoría económica como ley de rendimientos decrecientes).

Malthus y David Ricardo (1817) fueron los primeros que llamaron la atención sobre estos límites que el sector agrícola podría imponer al proceso de acumulación del capital. Para el primero, el crecimiento de la población generado por el incremento de los salarios, tiende a superar la capacidad de producción del sector agrícola necesaria para alimentar a toda la población. Mientras que para Ricardo la dificultad en el crecimiento no estaba en el aumento de la población, sino en la distribución de los recursos entre los diferentes sectores sociales. La tendencia a la baja de los beneficios industriales era la clave del freno del proceso de acumulación.

Schumpeter (1883-1950) se encargó de refutar las ideas que había dejado Malthus. Sostenía que el sistema capitalista generaba un nivel de competencia, que implicaba que los agentes deberían estar siempre innovando en sus procesos productivos. Por tanto, el resultado era un extraordinario crecimiento económico que superaría ampliamente al crecimiento de la población.

A mediados de siglo XX la teoría económica neoclásica se encontraba sin haber podido resolver el supuesto de los rendimientos decrecientes, lo cual traía una consecuencia devastadora acerca de la posibilidad de mantener el crecimiento económico en el tiempo.

De la mano del economista Solow, a partir del año 1956 los desarrollos teóricos neoclásicos encontrarán un nuevo punto de partida y se construirán en los fundamentos para dar comienzo a la teoría moderna neoclásica del crecimiento económico.

El modelo de Solow, incorpora los supuestos habituales del análisis clásico, como ser: el ahorro es siempre igual a la inversión, existencia de pleno empleo, competencia perfecta en los mercados, rendimientos decrecientes a escala y el capital y trabajo se suponen perfectamente sustituibles. En síntesis, en el largo plazo la única fuente de crecimiento 
económico es el progreso tecnológico3, pero como en todo modelo neoclásico, no es explicitado dentro del mismo, sólo asume la posición de una variable "exógena".

En la década del ochenta, desde el paradigma neoclásico se produce el aporte de Paul Romer que propone que el concepto de capital debe ser ampliado para incluir al capital humano- las habilidades y recursos productivos que incorpora el individuo a través de la educación, pasan a ser considerados como una inversión-, es decir, el conocimiento y la destreza de la mano de obra deben ser considerados como un factor de producción más. De esta manera, a diferencia del modelo de Solow, el crecimiento económico pasa a ser un componente endógeno que se determina dentro del modelo. Esto implicaría que el concepto de rendimientos marginales decrecientes no es aplicable al factor capital, lo que obtendrá con el tiempo son retornos crecientes, bajo la suposición de que se producirá una constante innovación tecnológica, generando rendimientos crecientes en la acumulación de capital.

La teoría del crecimiento endógeno del paradigma neoclásico asigna un papel importante al capital humano, supone que el conocimiento tiene la capacidad de generar nuevo conocimiento, garantizando retornos crecientes a escala y restando importancia a la apropiabilidad de ese conocimiento como fuente de poder del mercado.

Cabe destacar una gran fractura teórica que se produce dentro de las ciencias económicas, con la aparición de las ideas de Keynes (1883-1946). La teoría keynesiana (1936) obligaría a la teoría económica ortodoxa a reconocer la posibilidad del equilibrio con desempleo, en tanto, la escuela neoclásica se caracteriza por suponer la existencia de pleno empleo.

Entre el paradigma neoclásico y el keynesiano existe una contradicción entorno a los puntos centrales sobre el crecimiento económico, incluso se suelen utilizar las mismas variables pero invirtiendo el sentido de las causalidades.

Existen además muchas otras escuelas económicas que priorizan diferentes elementos como principales para conseguir un crecimiento económico de largo plazo.

Los factores que aseguran el crecimiento económico dentro de la teoría económica, siguen siendo un tema de acalorado debate, donde la tendencia no parece converger hacia una posición de consenso. 
En la actualidad la teoría del capital humano (Schultz, 1961), como solución propuesta por la teoría neoclásica para garantizar un crecimiento económico ilimitado, ha impregnado fuerte en el sentido común y representa la interpretación dominante. Sin embargo, no ha perdido vigencia como blanco de crítica.

Las críticas a los supuestos de la teoría del capital humano, se basan en varias concepciones y comprobaciones, entre las mas relevantes se encuentran: que la escolarización de la fuerza de trabajo no aumenta la productividad ni asegura la ejecución adecuada del puesto de trabajo; que el salario no depende de la escolaridad sino del puesto de trabajo ocupado en la jerarquía laboral; que el mercado de trabajo no opera sobre la base del libre juego de la oferta y la demanda de la fuerza de trabajo, sino que está sujeto a presiones políticas (empresariales y laborales).

Adelantándonos a lo que sigue a continuación, en base a los datos de las proyecciones poblacionales de Argentina para el periodo 2010-2040, podemos afirmar en términos demográficos que se tendría un fuerte impacto positivo sobre el sector educativo. El objetivo de este trabajo es poder cuantificarlo en términos económicos.

Pero afirmar lo anterior, es decir, poder expresar en términos económicos los cambios en la estructura de edad producto de la transición demográfica, solo es posible haciendo una serie de supuestos muy fuertes, que para nada se reflejan en la realidad. Estos nos permitirá poder hacer depender el gasto en educación de solamente los cambios en la estructura de edad de la población Argentina durante el periodo 2010-2040, producto de su transición demográfica.

Desde una perspectiva económica, para poder cumplir el objetivo de cuantificar el ahorro financiero que se producirá a lo largo del periodo 20102040, es necesario suponer que las siguientes variables permanecerán constantes, a los valores del año 2010 hasta el año 2040. Nos referimos a:

- las tasas de actividad y de empleo de la población de 14 años y más, por edades simples y sexo;

- la productividad de cada trabajador para cualquier edad y sexo;

- el comercio exterior y las transferencias financieras con el resto del mundo; $\mathrm{y}$

- los gastos en educación por alumno.

De esta manera podemos aislar el efecto puramente demográfico, del 


\section{Manzano - Impacto Bono Demográfico}

resto de las variables económicas vinculadas. Por tanto, la cantidad de alumnos, el valor agregado del sector educativo y el crecimiento del PBI, podrán proyectarse a partir de variables demográficas, es decir, sólo se encontrarán afectadas por los cambios en la estructura poblacional del periodo 2010-2040.

\section{El Efecto de la Transición Demográfica en Argentina sobre la Carga Demográfica}

Como mencionamos anteriormente, desde un enfoque puramente demográfico el crecimiento económico se relaciona con el periodo de tiempo durante el cual se produce un aumento de la población en edades potencialmente activas en relación a la población inactiva (o potencialmente dependiente).

En términos empíricos esta relación se expresa mediante el cálculo de la tasa de dependencia demográfica total ${ }^{4}$ (RDET).

En el caso de Argentina, la RDET presenta un descenso hasta el año 2035, momento en que finaliza el bono demográfico. La teoría demográfica considera todo este periodo de tiempo como económicamente favorable, dado que ofrece la posibilidad de aumentar las tasas de crecimiento económico per cápita y los niveles de bienestar de la población.

Con posterioridad al año 2035 la RDET comienza a aumentar, es decir, se eleva la carga demográfica sobre las personas en edades de trabajar (potencialmente productivas), enfrentando un periodo económicamente desfavorable.

Es importante aclarar, que hemos recortado a la población que asiste a un establecimiento educativo a las edades comprendidas entre 3 y 29 años, dado que el peso relativo de las que se encuentran de este fuera de este rango restante es marginal.

En el Cuadro $\mathrm{N}^{\circ} 1$, conforme se desarrolla la TTD en Argentina, durante el periodo 2010-2040 se produce un continuo descenso de la fecundidad-de 2,41 a 1,98 entre el año 2010 y 2040 - y un aumento en su esperanza de vida -de 75,3 años en el 2010 a 81,4 años en el año 2040-.

El valor de la RDET, que es resultado del cociente entre la población potencialmente dependiente -los menores de 14 años y los mayores de 65 años y más-, y las personas potencialmente activas (PET) - la población 
comprendida entre 14 y 64 años-, es de 56,7\% en el año 2010, desciende a 53,9 en el año 2035 -donde finaliza su descenso-, y luego se eleva a 54.7\% en al año 2040.

El incremento del valor de la RDET del año 2040, se debe a que la participación de la población adulta mayor -de 65 años y más-, supera al descenso de la población menor de 14 años sobre la población total, lo cual reduce la participación relativa de la PET sobre la población total.

\section{Tabla 1}

Indicadores Demográficos Seleccionados. Argentina, 2010-2040.

\begin{tabular}{|c|c|c|c|c|c|c|c|}
\hline Años & $\begin{array}{c}\text { Tasa } \\
\text { Global } \\
\text { de } \\
\text { fecundid } \\
\text { ad } \\
\text { (TGF) }\end{array}$ & $\begin{array}{c}\text { Esperanz } \\
\text { a de vida } \\
\text { al nacer } \\
\left(\mathbf{E}_{0}\right)\end{array}$ & $\begin{array}{l}\text { Relación de } \\
\text { dependenci } \\
\text { a de } \\
\text { menores de } \\
15 \text { años }\end{array}$ & $\begin{array}{c}\text { Relación de } \\
\text { dependencia de } \\
\text { mayores de } 65 \\
\text { años y más }\end{array}$ & $\begin{array}{c}\text { Relación } \\
\text { de } \\
\text { dependenc } \\
\text { ia } \\
\text { demográfi } \\
\text { ca }^{(*)}\end{array}$ & $\begin{array}{c}\text { Menores } \\
\text { de } 15 \text { años } \\
\text { sobre el } \\
\text { total } \\
\text { poblacion } \\
\text { al }\end{array}$ & $\begin{array}{c}\text { Mayores } \\
\text { de } 65 \text { años } \\
\text { y más } \\
\text { sobre el } \\
\text { total } \\
\text { poblacion } \\
\text { al } \\
\end{array}$ \\
\hline 2010 & 2,41 & 75,34 & 40,81 & 15,92 & 56,73 & 26,04 & 10,16 \\
\hline 2015 & 2,28 & 76,92 & 39,21 & 16,76 & 55,97 & 25,14 & 10,75 \\
\hline 2020 & 2,18 & 78,07 & 37,96 & 17,96 & 55,93 & 24,35 & 11,52 \\
\hline 2025 & 2,1 & 79,09 & 36,45 & 19,25 & 55,69 & 23,41 & 12,36 \\
\hline 2030 & 2,05 & 80,00 & 34,18 & 20,42 & 54,6 & 22,11 & 13,21 \\
\hline 2035 & 2,01 & 80,79 & 32,31 & 21,61 & 53,93 & 20,99 & 14,04 \\
\hline 2040 & 1,98 & 81,48 & 31,11 & 23,57 & 54,68 & 20,11 & 15,24 \\
\hline
\end{tabular}

(*)Relación de dependencia total = (población de menores de 15 años más la población de 65 y más años) / (población de 15 a 64 años) *100

Fuente: Elaboración propia en base a estimaciones y proyecciones elaboradas a partir de los resultados del Censo Nacional de Población, Hogares y Viviendas 2010.

También podemos expresar los resultados de la RDET de manera inversa, de modo que resulta más intuitivo. Es decir, como la relación entre los potenciales trabajadores entre los 14 y los 64 años (PET) y la población potencialmente inactiva (los menores de 14 años y los mayores de 65 años y más). Esta forma de expresar la carga demográfica, se la denomina tasa de sostenibilidad económica total (RSET).

Como vemos en el Cuadro $\mathrm{N}^{\circ} 2$, al igual que la RDET, el valor de la RSET se incrementa hasta el año 2035, cuando finaliza el bono demográfico 
y luego comienza a descender.

En el caso de la RSE de los mayores de 65 años y más, esta siempre es decreciente durante todo el periodo 2010-2040, debido a que se encuentra afectada por la tendencia al crecimiento de la esperanza de vida. Por el contrario, la RSE de la población menor de 14 años, presenta una tendencia de continuo ascenso, producto del efecto del descenso de la fecundidad durante todo el periodo 2010-2040.

Tabla 2

Relación de sostenibilidad. Argentina, 2010-2040.

\begin{tabular}{cccc}
\hline Años & $\begin{array}{c}\text { Relación de sostenibilidad } \\
\text { (con menores de 15 años) }\end{array}$ & $\begin{array}{c}\text { Relación de } \\
\text { sostenibilidad (con } \\
\text { mayores de 65 años) }\end{array}$ & $\begin{array}{c}\text { Relación de } \\
\text { sostenibilidad total }\end{array}$ \\
\hline $\mathbf{2 0 1 0}$ & 2,45 & 6,28 & 1,76 \\
$\mathbf{2 0 1 5}$ & 2,55 & 5,97 & 1,79 \\
$\mathbf{2 0 2 0}$ & 2,63 & 5,57 & 1,79 \\
$\mathbf{2 0 2 5}$ & 2,74 & 5,19 & 1,80 \\
$\mathbf{2 0 3 0}$ & 2,93 & 4,90 & 1,83 \\
$\mathbf{2 0 3 5}$ & 3,10 & 4,63 & 1,85 \\
$\mathbf{2 0 4 0}$ & 3,21 & 4,24 & 1,83 \\
\hline
\end{tabular}

Nota: Relación de sostenibilidad: RDS = P (14-64)/[P(0-14)+P(65+)].

Fuente: Elaboración propia en base a estimaciones y proyecciones elaboradas a partir de los resultados del Censo Nacional de Población, Hogares y Viviendas 2010.

\section{La Relación de Sostenibilidad Económica Calculada Utilizando la Información del Mercado de Trabajo y su Diferencia con el Cálculo Tradicional}

Hasta ahora hemos calculado la carga demográfica, teniendo en cuenta solo la dimensión demográfica. Es decir, toda la población comprendida entre 14 y 64 años era considerada como potenciales proveedores.

Pero sabemos que en realidad dentro de la franja etaria de 14 a 64 años, se encuentran personas desempleadas o inactivas. Y que también dentro del 
grupo poblacional de 65 años y más, se encuentran personas ocupadas dentro del mercado de trabajo.

A continuación calcularemos el valor de la relación de sostenibilidad económica (RSET), este caso consideramos como proveedores a todas las personas ocupadas de 14 años o más, y los consumidores netos reales, es la población comprendida por los menores de 13 años, más las personas mayores a 13 años que estén inactivos o desocupados.

En el Gráfico 1 vemos que la cantidad de ocupados total es de 18.645.609 trabajadores, de los cuales el $94,6 \%$ pertenece a la población entre 14 y 64 años - la cantidad de ocupados dentro de la PET es de 67,2\%, lo que es equivalente al valor de la tasa de empleo para la franja etaria de 14 a 64 años-, y el 5,4\% restante de los ocupados son personas de 65 años y más. La PET considerada anteriormente alcanza un total de 26.237 .824 personas, debido a que dentro de las mismas quedan incluidas 8.601 .035 de personas entre 14 y 64 años que no se encuentran ocupadas -1.146 .784 están desocupadas y 7.454.251 son inactivas-. En relación a la PET, la población de 14 años y más que se encuentra ocupada representa sólo el 71,1\% de la misma 


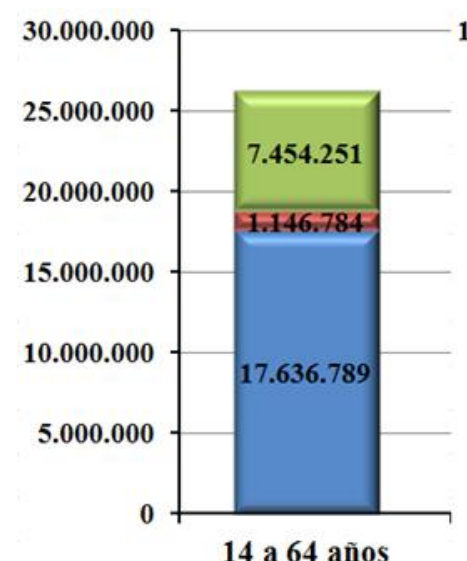

14 a 64 años

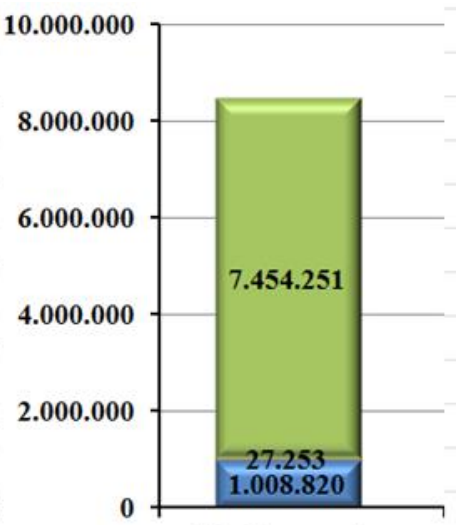

65 años y más

aPob. Ocupada a Pob. Desocupada $\square$ Pob. Inactiva

Figura 1. Condición de Actividad, según grupos de edad seleccionados. Argentina. Año 2010

Fuente: Elaboración propia en base los resultados del Censo Nacional de Población, Hogares y Viviendas 2010.

En la Tabla 3 presentamos los valores de los tres indicadores más representativos del mercado de trabajo para Argentina en el año 2010, diferenciando entre el grupo de 14 y 64 años y los adultos mayores (65 años y más).

En la población entre 14 y 64 años, algo más de 28 por cada 100 personas se encuentran inactivas, mientras que en el grupo de las personas de 65 años y más, el 74,0\% se encuentran inactivos. 
Tabla 3

Tasas relativas a la estructura laboral. Argentina, 2010.

\begin{tabular}{|c|c|c|c|c|c|c|c|c|c|}
\hline \multirow{2}{*}{$\begin{array}{l}\text { Edad en } \\
\text { grandes } \\
\text { grupos }\end{array}$} & \multicolumn{3}{|c|}{ entre 14 y 64 años } & \multicolumn{3}{|c|}{65 años y más } & \multicolumn{3}{|c|}{14 años y más } \\
\hline & $\begin{array}{c}\text { Tasa de } \\
\text { Inactividad }\end{array}$ & $\begin{array}{c}\text { Tasa de } \\
\text { Desocupación }\end{array}$ & $\begin{array}{l}\text { Tasa de } \\
\text { Empleo }\end{array}$ & $\begin{array}{c}\text { Tasa de } \\
\text { Inactividad }\end{array}$ & $\begin{array}{c}\text { Tasa de } \\
\text { Desocupación }\end{array}$ & $\begin{array}{l}\text { Tasa de } \\
\text { Empleo }\end{array}$ & $\begin{array}{c}\text { Tasa de } \\
\text { Inactividad }\end{array}$ & $\begin{array}{c}\text { Tasa de } \\
\text { Desocupación }\end{array}$ & $\begin{array}{l}\text { Tasa de } \\
\text { Empleo }\end{array}$ \\
\hline Argentina & 28,4 & 6,1 & 67,2 & 74,0 & 97,4 & 25,3 & 34,4 & 5,9 & 61,7 \\
\hline
\end{tabular}

Fuente: Elaboración propia en base a los resultados del Censo Nacional de Población, Hogares y Viviendas 2010.

En la Tabla 4 se presenta una comparación entre los valores de la RSET calculados de las dos maneras mencionadas.

Para el año 2010 la RSET según la relación puramente demográfica, presenta un valor de 1,76, mientras que el mismo indicador calculado teniendo en cuenta la situación del mercado laboral tiene un valor de sólo 0,87 ocupados por cada inactivo. La diferencia entre ambas, en todos los quinquenios del periodo 2010-2040, es bastante similar, presentando una brecha que oscila entre 0,86 y 0,90 puntos porcentuales. 
Tabla 4

Relación de sostenibilidad, según estructura demográfica y del mercado laboral. Argentina, 2010-2040.

Según relación puramente demográfica

Según situación del mercado laboral

\begin{tabular}{|c|c|c|c|c|c|c|}
\hline & $\begin{array}{c}\text { Población } \\
\text { potencialmente } \\
\text { Activa }\end{array}$ & $\begin{array}{c}\text { Población } \\
\text { potencialmente } \\
\text { Inactiva }\end{array}$ & RSET & $\begin{array}{l}\text { Población } \\
\text { Ocupada }\end{array}$ & $\begin{array}{c}\text { Población } \\
\text { Inactiva + } \\
\text { Desocupada }\end{array}$ & RSET \\
\hline 2010 & 26.024 .697 & 14.763 .756 & 1,76 & 18.929 .570 & 21.858 .883 & $\mathbf{0 , 8 7}$ \\
\hline 2015 & 27.653 .960 & 15.478 .006 & 1,79 & 20.304 .667 & 22.827 .299 & $\mathbf{0 , 8 9}$ \\
\hline 2020 & 29.101 .376 & 16.275 .387 & 1,79 & 21.598 .333 & 23.778 .430 & 0,91 \\
\hline 2025 & 30.491 .911 & 16.981 .849 & 1,80 & 22.801 .588 & 24.672 .172 & 0,92 \\
\hline 2030 & 31.959 .028 & 17.448 .237 & 1,83 & 23.964 .805 & 25.442 .460 & 0,94 \\
\hline 2035 & 33.247 .295 & 17.929 .792 & 1,85 & 25.035.199 & 26.141 .888 & 0,96 \\
\hline 2040 & 34.120 .730 & 18.657 .747 & 1,83 & 25.913 .243 & 26.865 .234 & 0,96 \\
\hline
\end{tabular}

Fuente: Elaboración propia en base a estimaciones y proyecciones elaboradas a partir de los resultados del Censo Nacional de Población, Hogares y Viviendas 2010.

Sin embargo, la tendencia es diferente. En el caso de la RSET que considera sólo la relación demográfica su trayectoria presenta un leve ascenso hasta el año 2035 -momento que alcanza su máximo de 1,85 dado que finaliza el bono demográfico-. Mientras que la RSET calculada incorporando la información del mercado laboral, presenta un suave crecimiento durante todo el periodo 2010-2040 -desde un valor de 0,87 en el año 2010 hasta a 0,96 en el año 2040-, es decir, un continuo descenso de la carga de dependencia demográfica en donde la existencia del bono demográfico pierde validez - debido a que estamos incorporando una variable que no es netamente demográfica- . 


\section{Estimación del Crecimiento del PBI en Base a Variables Demográficas}

La medida de la producción de riqueza de un país se expresa a través del indicador conocido como producto interno bruto (PIB). El indicador PIB per cápita, es el valor promedio del nivel de vida de la población, que como todo promedio posee la desventaja de no permitirnos conocer su nivel de dispersión. Cabe agregar que el PBI per cápita presenta una particularidad, debido que surge del cociente entre el PBI y la cantidad de población total, expresa de manera aritmética una relación entre la economía y la demografía.

Si se desea mantener el mismo valor de PBI per cápita, es necesario que el crecimiento de la riqueza sea igual al crecimiento poblacional. Dado que la tasa de crecimiento de la población tiende a disminuir en el tiempo, serán necesarios cada vez menores incrementos en la producción total del país.

A continuación realizaremos una estimación del PBI para el periodo 2015-2040, basada solo en los cambios en la estructura demográfica, para ello debemos tener presente todos los supuestos que hemos mencionamos en el Apartado II.

Realizaremos dos estimaciones del PBI -al igual que hicimos anteriormente con la RSET-, una netamente demográfica, utilizando para ponderar los cambios en la estructura demográfica de la PET. La segunda estimación será en función de los cambios en la estructura demográfica de la población de 14 años y más.

En la tabla se observan los resultados de ambas proyecciones del PBI nacional.

La estimación realizada utilizando la información del mercado laboral, presenta un crecimiento superior a la proyección del crecimiento económico basada en la dimensión puramente demográfica -los cambios en la estructura demográfica de la PET-. La tasa de crecimiento medio anual del periodo 2010-2040 es de 1,05 mientras que considerando la dimensión puramente demográfica es de 0,90. Ambas superan la tasa media de crecimiento anual poblacional del periodo $2010-2040$ que es de 0,86 por cada 1.000 habitantes. 
Tabla 5.

Proyección de la población total y del PBI pm, en función de la tasa de crecimiento anual de la PET y de la Población ocupada. Argentina. Año 2010-2040.

\section{Población Total}

\begin{tabular}{|c|c|c|c|c|c|c|}
\hline \multirow{2}{*}{ Año } & \multicolumn{2}{|c|}{ Población Total } & \multicolumn{2}{|c|}{$\begin{array}{l}\text { PBI }{ }_{\mathrm{PM}} \text {, según crecimiento } \\
\text { de la poblacional } \\
\text { potencialmente activa }\end{array}$} & \multicolumn{2}{|c|}{$\begin{array}{l}\text { PBI }{ }_{P M} \text {, según crecimiento de } \\
\text { la poblacional ocupada }\end{array}$} \\
\hline & Población & $\begin{array}{c}\text { Tasa de } \\
\text { crecimiento } \\
\text { medio anual }\end{array}$ & $\mathrm{PBI}_{\mathrm{PM}, 2004}$ & $\begin{array}{c}\text { Tasa de } \\
\text { crecimiento } \\
\text { medio anual } \\
\end{array}$ & $\mathrm{PBI}_{\mathrm{PM}, 2004}$ & $\begin{array}{l}\text { Tasa de } \\
\text { crecimiento } \\
\text { medio anual }\end{array}$ \\
\hline 2010 & 40.788 .453 & & 772.966 .580 & & 772.966 .580 & \\
\hline 2015 & 43.131 .966 & 1,12 & 821.357 .762 & 1,21 & 829.117 .019 & 1,40 \\
\hline 2020 & 45.376 .763 & 1,01 & 864.347 .857 & 1,02 & 881.942 .342 & 1,24 \\
\hline 2025 & 47.473 .760 & 0,90 & 905.648 .514 & 0,93 & 931.075 .820 & 1,08 \\
\hline 2030 & 49.407.265 & $\mathbf{0 , 8 0}$ & 949.223 .754 & 0,94 & 978.574 .440 & 1,00 \\
\hline 2035 & 51.177 .087 & $\mathbf{0 , 7 0}$ & 987.486 .921 & 0,79 & 1.022 .282 .694 & $\mathbf{0 , 8 7}$ \\
\hline 2040 & 52.778 .477 & 0,62 & 1.013 .429 .051 & 0,52 & 1.058 .136 .581 & 0,69 \\
\hline 2010-2040 & 11.990 .024 & 0,86 & 240.462.471 & 0,90 & 285.170.001 & 1,05 \\
\hline
\end{tabular}

Nota: Producto Interno Bruto a Niveles en miles de pesos a precios del año 2004.

Fuente: Elaboración propia en base a estimaciones y proyecciones elaboradas a partir de los resultados del Censo de Población 2010 y a información de la Dirección Nacional de Cuentas Nacionales (INDEC).

Vemos que en el caso de la proyección que considera solamente la dimensión demográfica, a partir del año 2035 presenta una tasa de crecimiento medio anual del PBI - de 0,52 en el año 2040-inferior a la del crecimiento de la población -de 0,62 en el año 2040-, por tanto, los niveles de producción del PBI per cápita comenzarían a disminuir a partir del año mencionado.

En cambio la proyección del PBI a partir de los cambios en la estructura etaria de las personas de 14 años y más -y su ponderación en base a tasas de empleo del año 2010-, muestra valores de crecimientos del PBI siempre superiores al crecimiento poblacional. Es decir, la producción per cápita presenta una tendencia al crecimiento durante todo el periodo en estudio. 
Esto se debe a que en la segunda estimación se está considerando a los ocupados de 65 años y más. Este grupo conforme avanza el envejecimiento de la estructura poblacional, tiene una mayor representación sobre la población total. Por tanto en relación a la PET, surge una ventaja adicional sobre el crecimiento económico, mediante el aporte que hace a la producción de riqueza el grupo de los adultos mayores.

En el apartado siguiente nos abocaremos a cuantificar en términos monetarios, los efectos del cambio en la estructura poblacional sobre el gasto en educación, según transcurre la transición demográfica en Argentina durante el periodo 2010-2040.

\section{La Cuantificación Económica del Cambio Demográfico en el Sector Educativo Argentino.}

Comenzaremos este apartado calculando la participación en términos económicos del servicio educativo en relación a la producción total de bienes y servicios de la economía del país.

Para ello es necesario tomar la información proveniente de la Dirección Nacional de Cuentas Nacionales (INDEC; $2015^{5}$ ), valor agregado del sector enseñanza -tanto público como privado- y valor agregado total de país -a precios del año 2004-.Considerando el valor promedio del periodo 2005 y 2010, el gasto educativo representa el 3,7 del valor agregado total de la economía, equivalente al 3,3\% del PBI nacional. El valor monetario promedio del sector enseñanza del periodo 2005-2010 asciende a 22.288.249 en miles de pesos a precios del año 2004. Ver Tabla 6 


\section{Manzano - Impacto Bono Demográfico}

Tabla 6.

Producto Interno Bruto y valor Agregado del Sector Enseñanza y del Total de la Economía. En miles de pesos a precios de 2004. Periodo 2005-2010.

\begin{tabular}{|c|c|c|c|c|c|c|c|}
\hline Año & 2005 & 2006 & 2007 & 2008 & 2009 & 2010 & $\begin{array}{r}\text { Promedio } \\
2005-2010 \\
\end{array}$ \\
\hline $\begin{array}{l}\text { VAB }_{\text {pb }} \\
\text { Sector }\end{array}$ & 19.831 .823 & 20.660 .595 & 21.734 .240 & 22.743 .344 & 23.674 .886 & 25.084 .607 & 22.288.249 \\
\hline $\begin{array}{c}\text { Enseñanza } \\
\text { VAB } \\
\text { Total de la } \\
\text { Economía }\end{array}$ & 513.175 .224 & 556.024 .196 & 598.846 .335 & 616.385 .548 & 615.731 .253 & 674.988 .386 & $\mathbf{5 9 5 . 8 5 8 . 4 9 0}$ \\
\hline $\begin{array}{c}\text { PBI }_{\text {pm Total }} \\
\text { de la } \\
\text { Economía } \\
(\%) \text { VAB pb } \\
\text { S. }\end{array}$ & 585.265 .574 & 634.283 .013 & 684.807 .291 & 705.864 .742 & 706.217 .847 & 772.966 .580 & 681.567 .508 \\
\hline $\begin{array}{c}\text { Enseñanza / } \\
\text { VAB pb } \\
\text { Total de la } \\
\text { Economía }\end{array}$ & 3,9 & 3,7 & 3,6 & 3,7 & 3,8 & 3,7 & 3,7 \\
\hline $\begin{array}{c}\text { (\%) VAB pb } \\
\text { S. } \\
\text { Enseñanza / } \\
\text { PBI pm } \\
\text { Total }\end{array}$ & 3,4 & 3,3 & 3,2 & 3,2 & 3,4 & 3,2 & 3,3 \\
\hline
\end{tabular}

Nota: VABpb (Valor Agregado Bruto a precios básicos); PIBpm (Producto Interno Bruto a precios de mercado)

Fuente: Dirección Nacional de Cuentas Nacionales (INDEC).

Procederemos a desagregar a la población entre 3 y 29 años que asiste a un establecimiento educativo en los siguientes niveles:

- Nivel Inicial: comprende a la población que asiste a un establecimiento educativo en las edades entre 3 y 5 años.

- Nivel Primario: la población que asiste a un establecimiento escolar comprendida entre 6 y 11 años -incluye al régimen primario y EGB-.

- Nivel Secundario: los jóvenes comprendidos entre 12 y 17 años que se encuentran asistiendo a un establecimiento escolar -incluye al régimen secundario y polimodal-, y

- Nivel Superior: conformado por la población comprendida entre los 18 y 29 años y que asiste a un establecimiento educativo de nivel superior incluye al nivel superior no universitario, universitario y post universitario-. 
Es necesario aclarar que dentro del mercado laboral se encuentran estudiantes, por tanto, un cálculo más refinado de las transferencias de ingresos hacia el sector educativo, sería excluyendo a la población entre 14 y 29 años que se encuentra asistiendo a un establecimiento educativo pero también se encuentra ocupada. Debido a esto, como venimos haciendo, realizaremos un cálculo alternativo que contemple también la situación real del mercado laboral.

En la Tabla 7 presentamos el porcentaje de asistencia según los cuatro niveles mencionados. Calculamos por un lado, todos los estudiantes entre 3 y 29 años que asisten a un establecimiento educativo, y por otro, consideramos solo al total de estudiantes que no se encuentran trabajando es decir, excluimos a los jóvenes entre 14 y 29 años, que se encuentran asistiendo a un establecimiento educativo pero además tienen una ocupación en el mercado laboral-. La diferencia entre ambas poblaciones estudiantiles en el año 2010 es de 10,9 p.p. en el porcentaje de asistencia en el nivel secundario y 14,2 p.p. en el nivel superior.

Tabla 7

Población que se encuentra asistiendo a un establecimiento educativo, según nivel. Porcentaje de asistencia. Año 2010.

\begin{tabular}{lccccc}
\hline Nivel Educativo & $\begin{array}{c}\text { Población } \\
\text { que } \\
\text { Asiste }\end{array}$ & $\begin{array}{c}\text { Población que } \\
\text { Asiste (excluidos } \\
\text { ocupados mayor } \\
\text { de 14 años) }\end{array}$ & $\begin{array}{c}\text { Población } \\
\text { Total }\end{array}$ & $\begin{array}{c}\text { Asistencia } \\
\text { total (\%) }\end{array}$ & $\begin{array}{c}\text { Asistencia } \\
\text { (excluidos } \\
\text { ocupados mayor } \\
\text { de 14 años) }(\%)\end{array}$ \\
\hline Nivel Inicial & 1.339 .860 & 1.339 .860 & 1.984 .070 & $\mathbf{6 7 , 5}$ & $\mathbf{6 7 , 5}$ \\
Nivel Primario & 4.062 .254 & 4.062 .254 & 4.104 .008 & $\mathbf{9 9 , 0}$ & $\mathbf{9 9 , 0}$ \\
Nivel Secundario & 3.752 .912 & 3.293 .606 & 4.215 .957 & $\mathbf{8 9 , 0}$ & $\mathbf{7 8 , 1}$ \\
Nivel Superior & 2.261 .043 & 1.144 .640 & 7.861 .295 & $\mathbf{2 8 , 8}$ & $\mathbf{1 4 , 6}$ \\
\hline
\end{tabular}

Fuente: Elaboración propia en base a los resultados del Censo de Población 2010.

En base a la información anterior sobre la participación según cada nivel del año 2010 -teniendo en cuenta los valores por edades simples y sexo-, estimaremos entonces la cantidad de alumnos según cada nivel, para el año 2020, 2030 y 2040. 
En la tabla 8 se observa que considerando todos los estudiantes entre 3 y 29 años, la proporción de la población estudiantil sobre el total poblacional en el cuadro anterior consideramos sobre su misma población en edad de estudiar-, era de $28,0 \%$ en el año 2010 , descendería en el 2020 a $26,7 \%$, a $24,8 \%$ en el año 2030 y se encontraría en $22,8 \%$ en el año 2040.

Realizando la proyección, pero excluyendo a los alumnos de entre 14 y más años que asisten a un establecimiento educativo y además se encuentran ocupados, la proporción de los mismos en relación a la población total era de $24,1 \%$ en el año 2010 y para las décadas siguientes esta descendería a: $23,1 \%$ en el año 2020; $21,4 \%$ en el año 2030 y $19,5 \%$ en el año 2040. 
Tabla 8

Proyección de la Población según nivel de asistencia y población total. En valores absolutos, proporciones y variaciones 2010-2020-2030 y 2040. Argentina.

Cantidad de estudiantes (suponiendo nivel de asistencia del 2010

Nivel

Educativo
Población que Asiste
Población que Asiste (excluidos ocupados mayor de 14 años)

\begin{tabular}{|c|c|c|c|c|c|c|c|c|}
\hline & 2010 & 2020 & 2030 & 2040 & 2010 & 2020 & 2030 & 2040 \\
\hline $\begin{array}{l}\text { Nivel Inicial } \\
\text { Nivel }\end{array}$ & 1.339 .860 & 1.514 .011 & 1.455 .964 & 1.425 .046 & 1.339 .860 & 1.514 .011 & 1.455 .964 & 1.425 .046 \\
\hline $\begin{array}{l}\text { Primario } \\
\text { Nivel }\end{array}$ & 4.062 .254 & 4.418 .155 & 4.346 .734 & 4.208 .626 & 4.062 .254 & 4.418 .155 & 4.346 .734 & 4.208 .626 \\
\hline $\begin{array}{l}\text { Secundario } \\
\text { Nivel }\end{array}$ & 3.752 .912 & 3.760 .796 & 3.997 .061 & 3.845 .485 & 3.293 .606 & 3.300 .525 & 3.507 .875 & 3.374 .849 \\
\hline $\begin{array}{l}\text { Superior* } \\
\text { Población }\end{array}$ & 2.261 .043 & 2.441 .147 & 2.463 .671 & 2.562 .129 & 1.144 .640 & 1.235 .817 & 1.247 .219 & 1.297 .063 \\
\hline $\begin{array}{c}\text { Total } \\
\text { Relación }\end{array}$ & 40.788 .453 & 45.376 .763 & 49.407 .265 & 52.778 .477 & 40.788 .453 & 45.376 .763 & 49.407 .265 & 52.778 .477 \\
\hline $\begin{array}{l}\text { Estudiantes/ } \\
\text { Pob. Total }\end{array}$ & 2010 & 2020 & 2030 & 2040 & 2010 & 2020 & 2030 & 2040 \\
\hline Nivel Inicial & 3,3 & 3,3 & 2,9 & 2,7 & 3,3 & 3,3 & 2,9 & 2,7 \\
\hline $\begin{array}{l}\text { Nivel } \\
\text { Primario }\end{array}$ & 10,0 & 9,7 & 8,8 & 8,0 & 10,0 & 9,7 & 8,8 & 8,0 \\
\hline $\begin{array}{l}\text { Nivel } \\
\text { Secundario }\end{array}$ & 9,2 & 8,3 & 8,1 & 7,3 & 8,1 & 7,3 & 7,1 & 6,4 \\
\hline $\begin{array}{l}\text { Nivel } \\
\text { Superior* }\end{array}$ & 5,5 & 5,4 & 5,0 & 4,9 & 2,8 & 2,7 & 2,5 & 2,5 \\
\hline Total & 28,0 & 26,7 & 24,8 & 22,8 & 24,1 & 23,1 & 21,4 & 19,5 \\
\hline
\end{tabular}

Nota: Educación Superior (incluye terciarios, universitarios y post universitarios).

Fuente: Elaboración propia en base a estimaciones y proyecciones elaboradas a partir de los resultados del Censo de Población 2010.

Vemos que para ambas proyecciones el peso relativo de la cantidad de estudiantes en relación al total poblacional tiende a decrecer en las tres décadas siguientes.

Para los niveles inicial y primario la disminución es la misma en las dos proyecciones. Pero se diferencian en el caso del nivel secundario y superior, por tanto la disminución total es diferente. Entre los años extremos, $2010 \mathrm{y}$ 2040, el porcentaje de población que asiste -excluidos ocupados mayores de 
14 años- sobre la población total desciende en $19,1 \%$, mientras que la población que asiste en relación al total de la población disminuye en 18,5\%.

Debido a que estamos suponiendo que tanto los niveles de cobertura escolar, como la tasa de empleo se mantienen constantes, la única variable que altera la proporción de alumnos que asisten a un establecimiento escolar en ambas proyecciones, es el cambio en la estructura demográfica, que se encuentra afectada por el efecto de la transición demográfica.

A continuación, para finalizar, estimaremos en términos monetarios, el efecto de la reducción de la participación de la población que asiste en relación al total poblacional.

En la Tabla 9 juntamos las proyecciones del PBI total calculadas anteriormente y adicionamos la proyección del valor agregado del sector enseñanza, diferenciando como venimos haciendo entre: resultados utilizando solo variables demográficas y considerando la situación en el mercado laboral.

En ambas proyecciones se observa que la participación del valor agregado del sector enseñanza como porcentaje del PBI total tiende a disminuir. 
Tabla 9

Gasto en Educación, expresado en términos de VAB en relación al Total del PBI. En miles de pesos a precios de 2004.

Considerando variables demográficas

Considerando los datos de asistencia educativa y de tasa de empleo del año 2010.

\begin{tabular}{|c|c|c|c|c|c|c|}
\hline Año & $\begin{array}{l}\text { VAB pb Sector } \\
\text { Enseñanza, } \\
\text { según } \\
\text { crecimiento } \\
\text { de la } \\
\text { Población que } \\
\text { Asiste. }\end{array}$ & $\begin{array}{l}\text { PBI }{ }_{\text {PM, según }} \\
\text { crecimiento de } \\
\text { la población } \\
\text { potencialmente } \\
\text { activa }\end{array}$ & $\begin{array}{c}\text { Relación } \\
\text { entre VAB } \\
\text { pb } S . \\
\text { Enseñanza } \\
\text { / PBI pm } \\
\text { Total }(\%)\end{array}$ & $\begin{array}{c}\text { VAB pb } \\
\text { Sector } \\
\text { Enseñanza, } \\
\text { según } \\
\text { crecimiento de } \\
\text { la Población } \\
\text { que Asiste } \\
\text { (excluidos los } \\
\text { ocupados } \\
\text { mayor de } 14 \\
\text { años). } \\
\end{array}$ & $\begin{array}{l}\text { PBI PM, } \\
\text { según } \\
\text { crecimiento } \\
\text { de la } \\
\text { poblacional } \\
\text { ocupada }\end{array}$ & $\begin{array}{c}\text { Relación } \\
\text { entre VAB pb } \\
\text { S. Enseñanza / } \\
\text { PBI pm Total } \\
(\%)\end{array}$ \\
\hline 2010 & 25.084 .607 & 772.966 .580 & 3,2 & 21.622 .291 & 772.966 .580 & 2,8 \\
\hline 2020 & 26.662 .363 & 864.347 .857 & 3,1 & 23.002 .525 & 881.942 .342 & 2,6 \\
\hline 2030 & 26.946 .521 & 949.223 .754 & 2,8 & 23.198 .710 & 978.574 .440 & 2,4 \\
\hline 2040 & 26.458 .399 & 1.013 .429 .051 & 2,6 & 22.644 .530 & 1.058 .136 .581 & 2,1 \\
\hline
\end{tabular}

Fuente: Elaboración propia en base a estimaciones y proyecciones elaboradas a partir de los resultados del Censo de Población 2010 y Dirección Nacional de Cuentas Nacionales (INDEC)

En el caso de proyectar considerando "solo variables puramente demográficas", la relación entre el valor agregado de la enseñanza en relación al PBI, se reduce de 3,2 en el año 2010 a 2,6\% en el 2040 -un $19,6 \%$ a lo largo de las tres décadas-. En términos monetarios sería un ahorro de algo más de 6.400 millones de pesos -a precios constantes del año 2004-. Mientras que en la proyección que considera "la situación en mercado laboral", en el año 2010 la relación entre el valor agregado del sector enseñanza y el PBI era de 2,8\%, y para el año 2040 la misma sería de solo $2,1 \%$, un disminución relativa de 23,5\% durante el periodo 2010-2040superior a la estimación anterior-. El ahorro es de algo más de 6.900 millones de pesos, a valores del año 2004. 
Los supuestos que hemos incorporado nos han permitido poder hacer depender las variables proyectadas en función de los cambios en las variables puramente demográficas, es decir las variaciones en el sector educativo y en la economía en su conjunto, se suceden conforme se producen modificaciones en la estructura de edad de la población Argentina durante el periodo 2010-2040, debido al avance de la transición demográfica.

En términos económicos, hemos comprobado que la evolución de la dinámica demográfica genera una situación favorable en el sector educativo de Argentina durante el periodo 2010-2040, mediante una disminución relativa del gasto total en la enseñanza. Este excedente podría volcarse a aumentar los niveles de cobertura del año 2010 o el gasto por alumno -o un mix de ambas políticas-.

A lo largo del trabajo hemos comparado los resultados de la estimación basada solo en la dimensión demográfica, con respecto a una proyección alternativa que incorpora también la situación del mercado laboral. Esta segunda estimación expresa un cálculo un tanto más refinado -o más realista-, los resultados presentan una situación aún más favorable en términos económicos, el descenso del gasto en educación en términos del PBI sería aún inferior y por tanto crecería el excedente durante todo el periodo hasta llegar el año 2040 -dado que el crecimiento económico se prolonga hasta finalizar nuestro periodo de estudio y no se encuentra afectado por el fin del efecto del bono demográfico en el año 2035-.

\section{Conclusiones}

Conforme avanza la transición demográfica se genera un periodo de descenso sostenido de la fecundidad, lo que implica una reducción de la población infantil y juvenil en términos relativos, mientras que aún no es muy grande el peso relativo de la población mayor de edad sobre el total poblacional y, por tanto, la principal proporción de población se concentra en las edades potencialmente activas.

Desde la teoría demográfica se propone que este periodo de tiempo, en el cual se produce un descenso de las relaciones de dependencia demográfica hasta alcanzar sus niveles más bajos -que se conoce como bono 
demográfico-, genera las condiciones para potenciar el crecimiento económico.

Sin embargo, incorporando algunos elementos de la teoría económica, se advierte que la condición necesaria para que tenga éxito el bono demográfico, sería logrando incrementar los niveles de las tasas de actividad y de empleo -sin que se produzcan descensos en los niveles de productividad-. Es decir, se debe garantizar un escenario de crecimiento económico, lo cual implicaría invertir la causalidad propuesta por los defensores del bono demográfico. De lo contrario, el periodo del bono demográfico generaría una presión sobre el mercado laboral, que se traduciría en un aumento del desempleo o en un descenso del nivel de los salarios -o cualquier combinación entre ambas-. En síntesis, el periodo del bono demográfico se transformaría es una restricción más al crecimiento económico.

A lo largo del desarrollo de las ciencias económicas no se ha avanzado sobre ninguna certeza acerca de cuáles son los factores determinantes respecto del crecimiento económico. En su desarrollo se destaca la proliferación de interpretaciones diversas y muchas veces contradictorias entre los diferentes paradigmas acerca del crecimiento económico. En síntesis, los factores que aseguran el crecimiento económico siguen siendo un tema de acalorado debate, donde la tendencia no parece converger hacia una posición de consenso.

En este trabajo utilizamos el enfoque de las Cuentas Nacionales de Transferencias (CNT), que nos permitió integrar las tendencias demográficas con ciertos instrumentos claves de la economía.

En base a información puramente demográfica, como son los datos de las proyecciones poblacionales de Argentina para el periodo 2010-2040, hemos podido cuantificar el impacto de la disminución de la carga de dependencia del sector educativo en términos económicos.

Para poder cumplir el objetivo propuesto, fue necesario realizar una serie de supuestos muy fuertes -que para nada se reflejan en la realidad-, permitiéndonos hacer depender la evolución del gasto en educación y del crecimiento del PBI, de solamente los cambios en la estructura de edad de la población Argentina durante el periodo 2010-2040, que se desenvuelve conforme se desarrolla la transición demográfica. 
En el apartado III, desde una perspectiva puramente demográfica, pudimos observar como la transición demográfica produce un descenso de la RDET hasta el año 2035, en donde se alcanza su menor valor $-53,9$ inactivos por cada 100 activos-. A partir del 2035, año en que finaliza el bono demográfico, la RDET comienza a ascender -el valor del año 2040 será de 54,7\%-, debido a que los incrementos de la población de 65 y más años sobre la población potencialmente activa, serán superiores a la reducción de la población menor a 14 años en relación a la PET.

La carga demográfica calculada considerando variables puramente demográficas, es un recorte que poco se asemeja a la realidad, dado que considera como potenciales proveedores a toda la población entre 14 y 64 años. Incorporando dentro de esta categoría a las personas que pertenecen a la franja etaria mencionada, pero que se encuentran desempleadas o inactivas. Al mismo tiempo excluye a todas las personas de 65 años y más que se encuentran ocupadas dentro del mercado de trabajo.

Por eso también hemos cálculado otro valor de la RSET alternativo, en base a los proveedores reales -las personas ocupadas de 14 años y más-, y a los consumidores netos reales -todos los menores de 14 años, más todos los mayores de 14 y más años que sean inactivos o desocupados-.

La diferencia en el valor del numerador de ambos RSET es significativa, la población de 14 años y más que se encuentra ocupada representa sólo el $71,1 \%$ de la PET, siendo la tasa de empleo promedio de los adultos mayores -65 años y más- de 25,3\%.

El valor de la RSET calculado considerando las variables únicamente demográficas presenta una superioridad con respecto al valor de la RSET que surge de ponderar la situación del mercado laboral, de una brecha que oscila entre 0,86 y 0,90 puntos porcentuales durante el periodo 2010-2040.

En el apartado V, hemos realizado una estimación del PBI basada solo en los cambios en la estructura demográfica, es decir, en función del avance de la transición demográfica.

Las proyecciones del PBI que hemos realizado consideró ambas alternativas: solo la dimensión demográfica, ponderando de acuerdo a los cambios en la estructura demográfica de la PET y teniendo en cuenta la modificación en la estructura demográfica de la población ocupada de 14 años y más. Los resultados que surgieron, muestran que la estimación del PBI realizada utilizando la información del mercado laboral, presentó un 
crecimiento superior

-TCMA de 1,05 durante el periodo 2010-2040- a la proyección del crecimiento económico basada en la dimensión puramente demográfica -TCMA de 0,86 durante estos 30 años-.

Ambas proyecciones considerando el periodo completo 20101-2040, presentan un valor de la tasa anual media de crecimiento anual del PBI, superior a la TCMA poblacional que sería de 0,86 por cada 1.000 habitantes, durante las tres décadas.

Sin embargo, analizando dentro del periodo 2010-2040, según años quinquenales a partir del año 2035, la proyección del PBI que considera solamente la dimensión demográfica, presenta una tasa de crecimiento medio anual del PBI - de 0,52 en el año 2040-inferior a la del crecimiento de la población -de 0,62 en el año 2040-, por tanto, en este caso los niveles del PBI per cápita comenzarían a disminuir a partir del año mencionado. Mientras que la proyección del PBI calculada a partir de los cambios en la estructura etaria de las personas ocupadas de 14 años y más, presenta valores de crecimientos del PBI siempre superiores al crecimiento poblacional durante el periodo 2010-2040.

En el último apartado, hemos calculado el descenso de la proporción de la población estudiantil sobre el total poblacional para el periodo 2010-2040. Para luego traducir este efecto en términos monetarios.

En el caso de la proyección que considera solo variables puramente demográficas, en el año 2010 el valor agregado del sector enseñanza representaba el 3,2\% del PBI, para el año 2040 este valor se reduciría a $2,6 \%$, lo que representa un ahorro de algo más de 6.400 millones de pesos -a precios constantes del año 2004-. Mientras que en la proyección que considera la situación en el mercado laboral, en el año 2010 el valor agregado del sector enseñanza en relación del PBI representaba el 2,8\%, y para el año 2040 esta será de solo 2,1\%, el ahorro es de algo más de 6.900 millones de pesos, a valores del año 2004.

Hemos comprobado en términos económicos, que la evolución de la dinámica demográfica, genera una situación favorable en el sector educativo de Argentina durante el periodo 2010-2040, mediante una disminución relativa del gasto del sector enseñanza, en relación al gasto total del país. Lo que genera un excedente económico que podría volcarse a aumentar los niveles de cobertura o el gasto por alumno -o un mix de ambas políticas-, en relación a la situación del año 2010. 


\section{Notas}

1 La transición demográfica es "el proceso del paso de elevados a bajos niveles de natalidad y de mortalidad” (Torrado, 1990; CEPAL-CELADE 1993)

2 La transición demográfica es "el proceso del paso de elevados a bajos niveles de natalidad y de mortalidad" (Torrado, 1990; CEPAL-CELADE 1993)

3 Lo que se conoce en la literatura económica como el "residuo de Solow"(Bernal: 2010)

4 La relación de dependencia total, es la relación entre las personas en edades inactivas respecto a las personas en edades activas. $\mathrm{Su}$ formulada de calculo es la siguiente: $(0-14)+$ (65 y más) / (15-64).

5 Sistema de consulta online de Agregados Macroeconómicos para los años 2004-2015 http://www.indec.gov.ar/nivel4_default.asp?id_tema_1=3\&id_tema_2=9\&id_tema_3=47

\section{Referencias}

Aguirre Pinto, G. (2011). El bono demográfico: una oportunidad de crecimiento económico. Revista Umbrales, 22, 157-173. Recuperado de: http://www.cides.edu.bo/webcides/images/pdf/Umbrales_22.pdf

Benítez Zenteno, R. (2004). Transición demográfica en América Latina. Tendencias y consecuencias sociales. Revista de Sociología, 66, 239254. Andras Uthoff

Botka, A.U. (2010). Transferencias intergeneracionales en América Latina: su importancia en el diseño de los sistemas de protección social. Comisión Económica para América Latina (CEPAL). Recuperado de:

http://repositorio.cepal.org/bitstream/handle/11362/3786/S2010653_ es.pdf? sequence $=1$

Castro Barrios, N. (2005). Análisis crítico de los enfoques sobre el crecimiento económico. Cuadernos Latinoamericanos, 16(27), 1145.

CEPAL/OIJ. (2008). Juventud y Cohesión Social en Iberoamérica. Un modelo para armar. Recuperado de: http://repositorio.cepal.org/bitstream/handle/11362/3639/S2008100_ es.pdf? sequence $=1$

Chackiel, J. (2000). El envejecimiento de la población latinoamericana: ¿hacia una relación de dependencia favorable?. Comisión Económica para América Latina (CEPAL/CELADE). Recuperado de: 
http://www.observatorionacionaldoidoso.fiocruz.br/biblioteca/_artigo s/92.pdf

Corrales Montoya, C. A. (1996). Consideraciones críticas respecto a la teoría neoclásica del crecimiento económico. Revista Universidad Eafit, 32,(104), 41-49. Recuperado de:

http://publicaciones.eafit.edu.co/index.php/revista-universidadeafit/article/viewFile/1172/1059

Diccionario Demográfico Multilingüe. (1959). Recuperado de: http://www.demopaedia.org/tools/spip.php?page=generate_dictionar y\&edition=es-i\&format=html

Instituto Nacional de Estadística y Censos (INDEC). Argentina: Censo Nacional de Población, Hogares y Viviendas2010. Recuperado de: www.indec.mecon.gov.ar.

Instituto Nacional de Estadística y Censos (INDEC). Estimaciones y proyecciones de población 2010-2040. Total del país. Serie de Análisis Demográfico, 35. Ciudad Autónoma de Buenos Aires, 2013. Recuperado de: www.indec.mecon.gov.ar.

Keynes, J.M. (1936). The General Theory of Employment, Interest and Money. Nueva York: Harcourt and Brace.

Malthus, T.R. ([1798]1970). Primer ensayo sobre la población. Madrid: Alianza.

Manzano, F. (2015). Bono demográfico y crecimiento económico en los países de América Latina. Un abordaje crítico e interdisciplinario (Tesis doctoral inédita). Universidad Nacional de Córdoba. Córdoba.

Pérez Díaz, J. (2011). Proyecciones de población; jóvenes y mayores. El futuro de la población, Información Estadística y Cartográfica de Andalucía, $\quad 1, \quad 217-223 . \quad$ Recuperado de: http://www.juntadeandalucia.es/institutodeestadisticaycartografia/Inf ormacionEstadisticayCartografica/RevistaFuturoPoblacion.pdf

Pérez Díaz, J. (2011). Reseña de "¿Revolución o declive demográfico? Una reflexión a partir del caso italiano" de Francesco C. Billari y Gianpiero Dalla Zuanna. Reis. Revista Española de Investigaciones Sociológicas, (134), 145-149. Recuperado de: http://redalyc.org/articulo.oa?id=9972226500

Roa, M.J. y Cendejas, J.L. (2007). Crecimiento económico, estructura de edades y dividendo demográfico. Documento de Trabajo del Centro 
de Investigación y Docencia Económicas (CIDE) de Méjico, SDTE 390. Recuperado de: http://www.libreriacide.com/librospdf/DTE390.pdf

Romer, P. (1986). Increasing Returns and Long-Run Growth. Journal of Political Economy, 94, 1002-1037. Recuperado de: http://www.parisschoolofeconomics.eu/docs/darcillon-thibault/paulromer-increasing-returns-and-long-run-growth.pdf

Saad, P.; Miller, T.; Martínez, C. y Holz, M. (2012). Juventud y bono demográfico en Iberoamérica. Comisión Económica para América Latina (CEPAL-OIJ-UNFPA). Recuperado de: http://repositorio.cepal.org/bitstream/handle/11362/1495/S2012103_ es.pdf? sequence $=1$.

Schultz, T. W. (1961). Investment in human capital. American Economic Review, 51(1), 1-17.

Schumpeter, J.A. (1963). Teoría del desenvolvimiento económico. Una investigación sobre ganancia, capital, crédito, interés y ciclo económico (3 ed.). México: Fondo de Cultura Económica.

Solow, R. M. (1956). A contribution to the theory of economic growth. Quarterly Journal of Economics, 70(1), 65-94.

Velázquez, G. A. (2010). Geografía y bienestar en la Argentina: Desigualdad de la sociedad y el territorio a comienzos del XXI. Geograficando, 6(6), 15-36.

Zavala de Cosío, M. E. (1995). Dos modelos de transición democrática en América Latina. Perfiles Latinoamericanos, 6, 29-47. Recuperado de: http://www.redalyc.org/articulo.oa?id=11560403

Fernando Manzano is professor at Universidad Nacional de Moreno and Universidad Provincial de Ezeiza (Argentina)

Contact Address: Direct correspondence to Fernando Manzano at Instituto de Geografía, Historia y Ciencias Sociales (CONICET/UNCPBA). Pinto 399 (7000) Tandil. Buenos Aires. Argentina. E-mail: fernandoarielmanzano@hotmail.com 